\title{
Elderly perform lower number of repetitions maximum than young at low instead high load resistance exercise
}

\author{
Amanda Veiga Sardelia,b*; Lucas do Carmo Santos ; \\ Wellington Martins dos Santosa; Cláudia Regina Cavaglieria; Mara Patrícia Traina Chacon-Mikahila.
}

\begin{abstract}
Background: Aging process is characterized by reduction of muscle mass and strength, named sarcopenia. To attenuate these declines, resistance training has been purposed. The use $1 \mathrm{RM}$ test has been applied to define the maximal strength and prescription of exercise. Otherwise, it is not safe and it has not shown good practical applicability. Thus, the present study aim to compare the repetitions number for high and low load resistance exercise performed until failure between a young and elderly men groups. Methods: We compared (Mann-Whitney test) the repetitions number performed until muscle failure by 9 young and 9 elderly men at four sets 45 일 press exercise for high (80\% 1RM) and low load (30\% 1RM). Results: Both groups reached maximum values for rate of perceived exertion, ensuring the muscle failure were achieved and no differences were seen between groups. The repetitions numbers were higher for young men with similar delta of reduction throughout sets between groups at low load resistance exercise. Otherwise, the delta of repetitions number reduction throughout sets at high load resistance exercise was higher for young men than elderly. Conclusions: We conclude elderly people need to perform lower number of repetition to reach muscle failure and thus achieve the desired training stimulus, mainly in low load resistance exercise. Furthermore, as they have better resistance to fatigue along sets the reduction of repetition number along sets is lower for them mainly when high load resistance exercise is performed.
\end{abstract}

Keywords: Resistance training; Exercise therapy; Exercise; Exercise tolerance; Health of the elderly.

\section{BACKGROUND}

Aging process is characterized by neuromuscular function and performance decline, mediated by the reduction in skeletal muscle mass and type II muscle fibers and its associated strength, known as sarcopenia ${ }^{(1,2)}$. Muscle strength capacity is primordial to keep good health, functional independence and avoid falls in aging ${ }^{(3-5)}$. Resistance exercise (RE) training has been purposed for this population ${ }^{(1,5,6)}$ to increase muscle mass and strength. The American College of Sports Medicine (ACSM) guidelines for exercise training recommended a training frequency of 2 to 3 days per week of RE, based on one repetition maximum (1RM) percentage for both young and older adults ${ }^{(5,7)}$.

To meet most the ACSM guidelines the use of $1 \mathrm{RM}$ test is necessary. Nevertheless, there are injuries risks through 1RM test, because of the requirement on the muscles, bone and ligaments ${ }^{(8,9)}$ and it may be enhanced in elderly who spend between $8-9$ sessions to determine the $1 \mathrm{RM}^{(9,10)}$. Furthermore, the use of individual's 1RM test seems to offer little practicality in the training routine due to the periodic adjustment necessary to prescribe the ideal training intensity ${ }^{(11)}$.
The RE performed until muscle failure even at low load is effective to increase muscle mass and strength to similar levels than traditional high load exercise based on 1RM percentage ${ }^{(12)}$. A good option to avoid these injuries risks is the RM range prescription widely used in the training practice. In fact, many authors have agreed that RE load prescription for increase in muscle strength and mass should be between 8 and 15 repetitions $(60-80 \%$ of $1-\mathrm{RM}){ }^{(13-15)}$.

The problem of the generalized applicability of the RM range suggested by previous researchers is that RM range varies among different populations. It is already known it varies between trained and untrained individuals, endurance and strength trained athletes, single-joint and multi-joint exercises, different muscle sizes, different exercises order, different RE intensities and we guess it varies also between young and elderly individuals $(7,16-20)$. Thus, to know the RM range for different populations and loads might be necessary for precise RE prescription.

The present study aimed to compare the RM range for high and low load resistance exercise between young and elderly

Correspondence: Amanda Veiga Sardeli. Laboratory of Exercise Physiology - FISEX, Faculty of Physical Education, University of Campinas, Av Érico Veríssimo, 701, ZIP code:13083-851, Campinas, São Paulo, Brazil, amandaveigasardeli@yahoo.com.br

aLaboratory of Exercise Physiology - FISEX, Faculty of Physical Education, University of Campinas (UNICAMP), Campinas - SP, Brazil

${ }^{b}$ Gerontology Program - Faculty of Medical Sciences, University of Campinas (UNICAMP), Campinas - SP, Brazil 
men groups. Furthermore we also compared the repetitions number reduction throughout sets between these groups. We hypothesized that elderly might have lower repetitions number for the same 1RM percentage and perhaps they will present higher magnitude of reduction, since they are usually weaker.

\section{METHODS}

\section{Sample}

We recruited 9 elderly and 9 young untrained healthy men. They were selected after a clinical evaluation, composed by anamnesis, rest and effort electrocardiogram by a cardiologist. Participants signed the informed consent approved by the Research Ethics Committee at University of Campinas (1.198.571) and the study was conducted in Physical Education Faculty in agreement with the statement of Helsinky 1975 (as revised in 2000).

At clinical evaluation, volunteers were excluded whether they had some pathology or other limitations which could enable them to practice exercise or could expose them to healthy risks. The limitations were: cardiopulmonary diseases, metabolic or pulmonary diseases, hypertension, osteoarticular diseases and obesity (body mass index $>30 \mathrm{~kg} / \mathrm{m}^{2}$ ).

\section{Experimental Design}

Following clinical evaluation participants underwent a familiarization session, 1RM test session and 1RM retest, all after at least $72 \mathrm{~h}$ of recovery. The volunteers performed the 2 RE sessions in a cross-over design with at least one week interval between them. They received instructions to rest/sleep well, stay $24 \mathrm{~h}$ without physical activities, without stimulators drinks (caffeine, etc) and alcohol, and attend the laboratory with $2 \mathrm{~h}$ fast.

Each participant performed every session at the same hour of the day (in the morning). They were instructed to follow the same diet in the days preceding both experimental sessions writing a $24 \mathrm{~h}$ food register (e.g.: medications, vitamin complex). Experimental sessions were occurred in the same room with controlled temperature between 21 and 23 degrees Celsius.

\section{One repetition maximum test (1RM)}

$1 \mathrm{RM}$ test was performed to assessment of maximum strength on leg press exercises performed on leg press equipment (Nakagym, Brasil), according to descriptions by Brown et al.(21). Subjects cycle for 5 minutes for general warm-up at a cycle ergometer. Sequentially, they performed 8 repetitions at $50 \%$ of their estimated 1RM (according to each participant's capacity and 3 repetitions at $70 \%$ of their estimated 1RM, for specific warm-up. Following warm-up, $1 \mathrm{RM}$ trials were performed with progressively heavier weights until the 1RM was determined within 5 attempts, with 3-5 min of rest between trials. We registered the highest load value obtained after the test-retest for RE protocols prescription.

\section{Rate perceived exertion}

The Rate of perceived exertion (RPE) was applied to evaluate whether the participants really performed exercise sets until failure. The 6-20 points Borg Scale was shown at the end of each set including the warm-up set.

\section{Experimental protocols}

Experimental protocols were performed at 450 leg press equipment (Nakagym, São Paulo, Brasil). For warm up, both protocols were preceded by 10 repetitions with 30\% 1RM, and following by the 4 sets until voluntary failure, intercalated by 1 min rest interval. High (HL) and low load (LL) protocols were performed at $80 \%$ and $1 \mathrm{RM}$ and $30 \% 1 \mathrm{RM}$, respectively.

\section{Statistical treatment}

According to Shapiro-Wilk test, data were not normally distributed. We applied Mann-Whitney $U$ test for group comparisons (young and elderly) within the same RE protocol. We also compared number of repetitions delta of changes between groups Mann-Whitney $U$ test respectively. In all cases the $p \leq 0.05$ was adopted as statistical significance level. All data are presented as median $\left(25^{\text {th }} ; 75^{\text {th }}\right)$. The software PASW statistic 18.0 (SPSS Inc., Chicago, USA) was used for statistical analyzes.

\section{RESULTS}

The subjects and protocols features are described in table 1. As expected the young men had higher strength $1 \mathrm{RM}$ than elderly. We followed the RPE along RE protocols to ensure the maximal effort in all sets; therefore, there was no difference for RPE between groups (Figure 1).

There was no significant difference between young and elderly for $\mathrm{HL}$ repetitions number (Table 2). Otherwise, for $\mathrm{LL}$, young men performed higher repetitions number than elderly at first set and considering the total repetitions number. Furthermore, the apparent reduction of repetitions number magnitude along sets for young men were statistically higher than elderly from $2^{\text {nd }}$ to $3^{\text {rd }}$ set within $\mathrm{HL}$ protocol (Table 2 ).

\section{Ratio perceived exerction (RPE)}

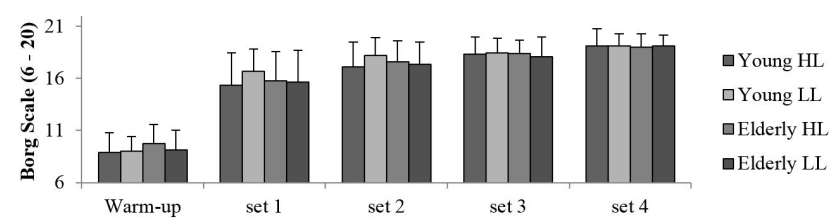

Figure 1. Ratio perceived exertion (RPE) during the warm-up and HL and LL sets for young and elderly men. Legend: HL: High Load; LL Low Load. 
Table 1. Subjects and protocols features.

\begin{tabular}{|c|c|c|c|}
\hline & Young & Elderly & $P$ value \\
\hline Age (years) & $27(24 ; 29)$ & $63(62 ; 64)$ & $<0.001$ \\
\hline Weight (kg) & $67.6(59 ; 72)$ & $72.8(68 ; 80)$ & 0.04 \\
\hline Height $(\mathrm{cm})$ & $1.73(1.68 ; 1.76)$ & $1.69(1.64 ; 1.74)$ & 0.19 \\
\hline $\mathrm{BMI}\left(\mathrm{kg} / \mathrm{cm}^{2}\right)$ & $22.3(21.1 ; 23.3)$ & $26.4(24.4 ; 27.2)$ & $<0.001$ \\
\hline $1 \mathrm{RM}(\mathrm{kg})$ & 350 (292: 412) & $210(200 ; 260)$ & 0.005 \\
\hline \multicolumn{4}{|l|}{ HL } \\
\hline Exercise duration (s) & $415(402 ; 473)$ & $438(411 ; 475)$ & 0.47 \\
\hline Exercise Load (Kg) & $240(218 ; 330)$ & $168(160 ; 208)$ & 0.008 \\
\hline \multicolumn{4}{|l|}{ LL } \\
\hline Exercise duration (s) & $515(40.5 ; 586)$ & $575(509 ; 602)$ & 0.40 \\
\hline Exercise Load (Kg) & $99(81 ; 123)$ & $63(60 ; 78)$ & 0.007 \\
\hline
\end{tabular}

Legend: Data are presented as median (25 $\left.; 7^{\text {th }}\right)$. BMI: Body mass index; 1RM: One repetition maximum; HL: High Load; LL: Low Load.

Table 2. Comparison for repetitions number at each set between young and elderly men.

\begin{tabular}{|c|c|c|c|}
\hline & Young & Elderly & $P$ value \\
\hline \multicolumn{4}{|l|}{ HL } \\
\hline \multicolumn{4}{|c|}{ Repetitions number } \\
\hline set 1 & $16(11 ; 18)$ & $13(10.5 ; 15)$ & 0.28 \\
\hline set 2 & $13(9 ; 15)$ & $10(8.5 ; 11.5)$ & 0.11 \\
\hline set 3 & $8(6.5 ; 10)$ & $10(8 ; 10)$ & 0.20 \\
\hline set 4 & $8(4 ; 9)$ & $9(6.5 ; 10.5)$ & 0.16 \\
\hline Total & $43(31 ; 50)$ & $40(34.5 ; 48)$ & 0.92 \\
\hline \multicolumn{4}{|c|}{ Delta of change between sets } \\
\hline Set 1 to Set 2 & $4(3 ; 5)$ & $3(2 ; 4)$ & 0.89 \\
\hline Set 2 to Set 3 & $3(2 ; 6)$ & $0(-2 ; 2)$ & $<0.001$ \\
\hline Set 3 to Set 4 & $1(0 ; 3)$ & $0(-1 ; 2)$ & 0.62 \\
\hline \multicolumn{4}{|l|}{ LL } \\
\hline \multicolumn{4}{|c|}{ Repetitions number } \\
\hline set 1 & $62(53 ; 69)$ & $44(40.5 ; 54)$ & $<0.001$ \\
\hline set 2 & $33(24.5 ; 40.75)$ & $27(20.5 ; 31)$ & 0.11 \\
\hline set 3 & $21.5(18,5 ; 25,25)$ & $24(18.5 ; 26)$ & 0.73 \\
\hline set 4 & $19(15.5 ; 26.75)$ & $19(17.5 ; 20)$ & 0.96 \\
\hline Total & $131(126 ; 151)$ & $109(99 ; 131)$ & 0.05 \\
\hline \multicolumn{4}{|c|}{ Delta of change between sets } \\
\hline Set 1 to Set 2 & $27(22 ; 40)$ & $21(14 ; 24)$ & $<0.001$ \\
\hline Set 2 to Set 3 & $8(4 ; 9)$ & $2(-1 ; 8)$ & 0.09 \\
\hline Set 3 to Set 4 & $1(0 ; 5)$ & $2(0 ; 8)$ & 0.88 \\
\hline
\end{tabular}

Legend: Data are presented as median $\left(25^{\text {th }} ; 75^{\text {th }}\right)$. HL: High Load; LL Low Load.

\section{DISCUSSION}

The main findings of the present study were (1) higher repetitions number for young than elderly men along LL sets and (2) higher decline in repetitions number along HL sets for young compared to elderly men.
It is noteworthy ACSM ${ }^{(7)}$ recommend higher repetitions number for elderly (10-15) compared to young (8-12). In this way, it seems they recommended lower load for elderly, as seeing they would not perform higher repetitions number than young for the same load. 
In the other hand the 8-12 repetitions purposed for young could be too low for them, since the untrained young men of the present study reached a median of 16 repetitions in the first set. Meantime, the problem of these divergences is not the RM repetitions range number, since variations of 2 to 5 repetitions will be seeing among individuals, without losses in the muscle adaptations, considering the importance of require complete fiber recruitment ${ }^{(22)}$. Those findings call attention for a new matter of debate: the combination of volume and load to reach maximal effort is more important than only load and the achievement of RE until failure should be recommended.

The main limitation of the RM range prescription is the neglected number of repetition reductions along sets. Although both age groups reduced repetition number throughout the sets, the reduction magnitude for young men was higher. Kent-Braun ${ }^{(23)}$, already discussed about the increase in muscle resistance to fatigue with age. This phenomenon might be due to many factors, such as: (1) lower maximal motor unit discharge rates seeing in central nervous system; (2) a potentially shift to increase type I fibers, which arises from type II motor units loss and consequential reinnervation of muscle fibers by type I motor neurons (3).

Someone might believe the higher repetitions number reduction in young men, is due to higher performance at first sets, while elderly try to preserve themselves; however it is denied by the RPE values, that do not differed between young and elderly men.

In this way, some researches have postulated the determinacy of failure repetitions number achievement to induce muscle adaptations ${ }^{(12,22,24)}$. We showed elderly people need lower repetitions number to reach failure for $L L$, which means the exercise prescription for elderly men should not be based on the same RM range than young men. Thus, exercise prescribers might be aware of the different muscle potentials (regarding RM number in each set and reduction along sets) between young and elderly in both RE loads.

\section{Limitations}

A present study limitation was the investigation of only lower limb exercise, since the tolerance to endure might be different for each muscle group. Otherwise, we chose lower limb resistance exercise because it has been shown to be enough to keep good health, functional independence and avoid falls in aging ${ }^{(3-5)}$. The second limitation was the time execution control for concentric and eccentric phases. Despite they have received orientation for $1 \mathrm{~s}$ execution during each phase, some of than could not perform to fast and it could be a confusing factor for our findings. Anyway, we believe the cross over design is enough to keep the between individuals difference nulled.

\section{CONCLUSIONS}

We showed the RE prescription based on RM range should be different between young and elderly, with higher repetition numbers for young rather than elderly men when low load is desired. Regarding high load RE the repetitions numbers along sets are not too different between young and elderly. The reduction of repetitions number throughout high load RE sets had higher magnitude for young than elderly, thus, we conclude the RM range prescription have to be different for each exercise set and a steep reduction of repetition number for young have to be prescribed.

\section{AUTHORS' CONTRIBUTIONS}

AVS, LCS, WMS, AFG and MPTC developed the experimental design. AVS, LCS and MLVF carried out the data collection. AVS, LCS, AFG and WMS performed data analysis. AVS, LCS and WMS conceived the manuscript that was reviewed for AFG, MVH, CRC and MPTC.

\section{COMPETING INTERESTS}

Nothing to declare.

\section{REFERENCES}

1. Marimuthu K, Murton AJ, Greenhaff PL. Mechanisms regulating muscle mass during disuse atrophy and rehabilitation in humans. Journal of applied physiology. 2011;110:555-60.

2. Doherty TJ. Invited review: Aging and sarcopenia. Journal of applied physiology. 2003;95:1717-27.

3. Reid KF, Naumova EN, Carabello RJ, Phillips EM, Fielding RA. Lower extremity muscle mass predicts functional performance in mobilitylimited elders. The journal of nutrition, health \& aging. 2008;12:493-8.

4. Ishigaki EY, Ramos LG, Carvalho ES, Lunardi AC. Effectiveness of muscle strengthening and description of protocols for preventing falls in the elderly: a systematic review. Brazilian journal of physical therapy. 2014;18:111-8.

5. ACSM. American College of Sports Medicine position stand. Progression models in resistance training for healthy adults. Medicine and science in sports and exercise. 2009;41:687-708.

6. Reeves ND, Narici MV, Maganaris CN. In vivo human muscle structure and function: adaptations to resistance training in old age. Experimental physiology. 2004;89:675-89.

7. Nelson ME, Rejeski WJ, Blair SN, Duncan PW, Judge JO, King AC, et al. Physical activity and public health in older adults: recommendation from the American College of Sports Medicine and the American Heart Association. Medicine and science in sports and exercise. 2007;39:143545.

8. Braith RW, Graves JE, Leggett SH, Pollock ML. Effect of training on the relationship between maximal and submaximal strength. Med Sci Sports Exerc. 1993;25:132-8.

9. Ploutz-Snyder LL, Giamis EL. Orientation and familiarization to $1 R M$ strength testing in old and young women. Journal of strength and conditioning research / National Strength \& Conditioning Association. 2001;15:519-23.

10. Niewiadomski W, Laskowska D, Gasiorowaska A. Determin ation and prediction of one repetition maximum (1RM): safety considerations. J Hum Kinet. 2008;19:109-20

11. Fleck SJ, Kraemer WJ. Disigning Resistance Training Programs. Champaign: Human Kinetics; 1997. 
12. Mitchell CJ, Churchward-Venne TA, West DW, Burd NA, Breen L, Baker SK, et al. Resistance exercise load does not determine training-mediated hypertrophic gains in young men. Journal of applied physiology. 2012;113:71-7.

13. American College of Sports M. American College of Sports Medicine position stand. Progression models in resistance training for healthy adults. Medicine and science in sports and exercise. 2009;41:687-708.

14. VERKHOSHANSY Y, SIFF MC. Supertraining. 6ed ed2009.

15. BOMPA TO, CORNACCHIA L. Musculação Treinamento de Força Consciente. São Paulo: Phorte Editora; 2000.

16. Richens $B$, Cleather DJ. The relationship between the number of repetitions performed at given intensities is different in endurance and strength trained athletes. Biology of sport. 2014;31:157-61.

17. Alves $B H$, Roberto $S$, Dias MR. Número de repetições e percentual de cara máxima: Comparação entre exercício uni e multiarticular. Revista Brasileira de Prescrição e Fisiologia do Exercício2012.

18. Simão R, Poly MA, Lemos A. Prescrição de exercícios através do teste de uma repetição máxima (T1RM) em homens treinados. Fitness \&amp; Performance Journal2004. p. 47-52.
19. Arazi $H$, Asadi A. The relationship between the selected percentages of one repetition maximum and the number of repetitions in trained and untrained males. Physical Education and Sport2011. p. 25 - 33.

20. Moraes E, NP M, Maia M, BF S, H M, R. S. Influence of exercise order on the number of repetitions in untrained teenagers. MTP \& Rehab Journal; 2016.

21. Brown LE, Weir JP. Procedures recommendation I: Accurate assessment of muscular strength and power. Journal of Exercise Physiology Online. 2001;4:1-21.

22. Burd NA, Mitchell CJ, Churchward-Venne TA, Phillips SM. Bigger weights may not beget bigger muscles: evidence from acute muscle protein synthetic responses after resistance exercise. Applied physiology, nutrition, and metabolism = Physiologie appliquee, nutrition et metabolisme. 2012;37:551-4.

23. Kent-Braun JA. Skeletal muscle fatigue in old age: whose advantage? Exercise and sport sciences reviews. 2009;37:3-9.

24. Barcelos LC, Nunes PR, de Souza LR, de Oliveira AA, Furlanetto R, Marocolo $M$, et al. Low-load resistance training promotes muscular adaptation regardless of vascular occlusion, load, or volume. European journal of applied physiology. 2015;115:1559-68. 